\title{
Electronic structure, doping, order and disorder in cuprate superconductors.
}

\author{
T. Jarlborg \\ DPMC, University of Geneva, 24 Quai Ernest-Ansermet, \\ CH-1211 Geneva 4, Switzerland
}

\begin{abstract}
The electron-phonon and spin-phonon coupling in typical high- $T_{C}$ cuprates, like LSCO and HBCO are peaked for just a few q-vectors because of the 2-dimensional Fermi surface shape. The activation of few spin-phonon modes compensates for the low electronic density-of-states, and the superconducting $T_{C}$ can be high. Thermal disorder of the lattice perturbs the strongly coupled modes already at moderately high temperature. This happens because of incoherent potential fluctuations of the Madelung term and reduced spin-phonon coupling. This effect puts a limit on long-range superconductivity, while fluctuations can persist on a shorter length scale at higher temperatures. BCS-type model calculations are used to show how disorder can reduce the superconducting gap and $T_{C}$. Ordering of dopants into stripes has been suggested to improve superconducting properties, mainly through an increased DOS. Such a mechanism seems to be a good way to make $T_{C}$ more resistant to thermal disorder at low doping.
\end{abstract}

PACS numbers: 74.20.Pq,74.72.-h,74.25.Jb

\section{INTRODUCTION.}

Calculations of the electron-phonon coupling $\lambda$ (EPC) 1] can be made from band parameters coming from selfconsistent electronic structure results based on densityfunctional theory (DFT) 2,4]. In this formalism we write the EPC as

$$
\lambda=N\left(E_{F}\right) I^{2} / M \omega^{2}
$$

where $N\left(E_{F}\right)$ is the density-of-states (DOS) at the Fermi energy, $M$ is an atomic mass, $\omega$ is the averaged phonon frequency $\left(M \omega^{2}\right.$ is a force constant $K=\partial^{2} E / \partial u^{2}$, the second derivative of the total energy, $E$, with respect to the atomic displacement $\partial u^{2}$ ) and $I$ is the matrix element $\left\langle\frac{\partial V(r)}{\partial u}>\right.$ evaluated at the $E_{F}(V(r)$ is the electron potential). I can be determined from the change in electron energies, $\epsilon_{k}$, in "frozen" phonon calculations [5, 6]. Such calculations show generally that $I$ is large only when $k$ is close to $\kappa$, the momentum for the phonon. The matrix element is constant for harmonic vibrations, $I_{k}=\left(\epsilon_{k}^{u}-\epsilon_{k}^{0}\right) / u=\vartheta_{k} / u$. The same formalism can be applied for coupling to spin fluctuations (SF) essentially by replacing displacements $u$ by magnetic moments $m$ [7, 8.

Most doped cuprates have very simple Fermi surfaces (FS), and retaining that $I$ is large only for $q=\kappa$, allow us to calculate most of the coupling from a few phonon distortions only [6]. Furthermore, by rewriting the BCS equation for $T_{C}[9]$,

$$
k_{B} T_{C}=1.13 \hbar \omega e^{-1 / \lambda}
$$

as 10, 11; ;

$$
K u^{2}=N \vartheta^{2} \ln \left(1.13 \hbar \omega / k_{B} T_{C}\right)
$$

it becomes clear that superconductivity is possible when the cost in total vibrational energy $\left(K u^{2}\right)$ of participating phonons is smaller than a fraction (depending on the $l n$ function) of the gain in electron energy $\left(N \vartheta^{2}\right)$. Then, for a simple $\mathrm{FS}$, it is possible to have a large $T_{C}$ despite a low value of $N\left(E_{F}\right)$, because the energy cost from excitations of only a few phonons will be small [11. Phonons, spinfluctuations and charge-waves are all possible excitations for activation of superconductivity, but only if the electronic energy gain can overcome the excitation energy. For conventional superconductors we usually ignore everything except phonons when $T_{C}$ is to be estimated. Here, for cuprates it is more complicated, since we assume that only part of the phonon- and spin-fluctuation spectra should be counted. Internal coupling within the simple FS opens the possibility for $d$-wave superconductivity depending on the strength of electron-electron repulsion [12].

From such arguments we claim that the normally estimated couplings to phonons and spin fluctuations can explain large $T_{C}$ 's, at least quantitatively. Lattice deformations can influence magnetism and other T-dependences of physical properties [13. In the cuprates this leads to spin-phonon coupling (SPC), where phonon distortions will enforce anti-ferro magnetic (AFM) excitations and vice-versa (and $\lambda$ ) [6]. Resonant inelastic X-ray scattering experiments show indications of coupling between magnetic and lattice modes [14]. Thus, SPC might be important for high- $T_{C}$ superconductivity. But the constructive SPC in the cuprates will degrade at large $\mathrm{T}$ because of disorder, and if superconductivity is mediated by few SPC modes only, it is probable that superconductivity will suffer at high $T$ [15]. Superconductors with complicated FS's and multiple phonon modes, like transition metals and their compounds, do not have this problem, partly because SPC is not likely to be important, but also because $T_{C}$ is not very high. The simulation of EPC and SPC by frozen phonon- and spin-wave calculations opens the possibility how sensitive the superconductivity carrying modes are to lattice disorder. Here we will 
include thermal disorder and zero-point motion (ZPM) in the lattice together with the phonons and spin waves in order to see how the waves, band gaps, coupling and $T_{C}$ are modified. Thermal disorder is shown to be important for ground state properties [16 22 and spectroscopic responses 23 27] in different materials. Even ZPM is important for some properties, and since $T_{C}$ can be of the order $100 \mathrm{~K}$ in cuprates we approach a regime where band broadening becomes large in comparison to superconducting gaps and peaks in the density-of-state (DOS). This will be investigated below for $\mathrm{La}_{(2-n)} \mathrm{Ba}_{n} \mathrm{CuO}_{4}$. Finally we also present preliminary results of how ordering of impurities into stripes can be useful to diminish the bad effects of disorder on $T_{C}$ [28, 32].

\section{METHOD OF CALCULATION.}

The band structures are calculated by the linear muffin-tin orbital (LMTO) method 33, 34 and the local spin-density approximation (LSDA) 35. Supercells extending 2 lattice constants $\left(a_{0}\right)$ along $\vec{y}$ and $8 a_{0}$ along $\vec{x}$ contain 112 atoms totally, see Fig. 11. The doping $n=0.125(0.25$ holes per $\mathrm{Cu})$ (needed to assure that waves along this cell makes the gap at $E_{F}$ ) are taken into account in the virtual lattice approximation by using nuclear and electronic charges on La equal to 56.875 . The spin-polarized calculation include staggered fields of strengths up to $\pm 0.33 \mathrm{eV}$ on $\mathrm{Cu}$ sites in order to induce anti-ferro magnetic (AFM) stripes on the $\mathrm{Cu}$ lattice separated by one layer of non magnetic $\mathrm{Cu}$, see Fig. 1 .

Planar oxygens are displaced by a maximum of $0.07 \AA$ in the calculations with an O-"breathing" phonon mode. This displacement amplitude $u_{O}$ (which corresponds to thermally activated phonons at room temperature, RT) is large enough to obtain precise values of $\vartheta$ and $I$ in the harmonic approximation. The results for cells having phonon- and spin-waves show a general enforcement of both waves, and enhanced coupling parameters, as shown previously [6]. In addition, the frozen phonon calculations show that $\vartheta_{k}$ is large near $k=Q-q$ ( $Q$ defines the AFM on near neighbor $\mathrm{Cu}$ sites, and $q$ defines the long-range modulation), and very small elsewhere. The calculations for ordered cells or with frozen phonons will be complemented by other sets of supercells, where all atomic positions are displaced randomly with average displacements $u$, in order to simulate thermal disorder.

\section{RESULTS AND DISCUSSION.}

\section{A. Bands and coupling constants.}

The cuprate FS's are 2-dimensional cylinders. The FS becomes almost diamond shaped at optimal doping, when the FS reaches the X-point of the Brillouin Zone (BZ) and the DOS has a van-Hove singularity peak [15]. The calculated coupling strength for phonon/spin waves

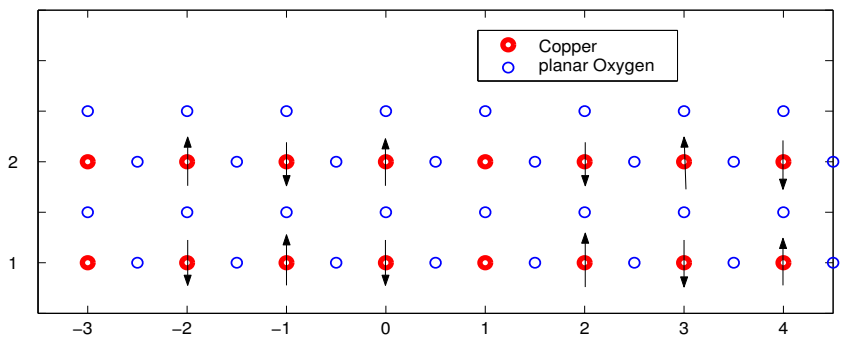

FIG. 1: (Color online) Schematic plot of the $\mathrm{CuO}$ plane of the unit cell. The arrows indicate the applied magnetic field on some $\mathrm{Cu}$ atoms that leads to AFM stripes.

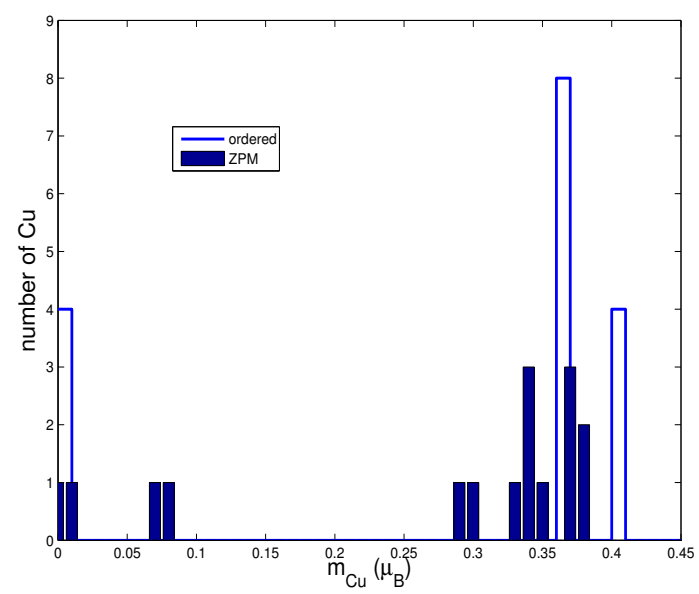

FIG. 2: (Color online) The distribution of the absolute values of the local $\mathrm{Cu}$ moments for ordered and ZPM-disordered structures within supercells as in Fig. 1. The applied field on 12 of the $16 \mathrm{Cu}$ sites is $\pm 0.33 \mathrm{eV}$ in both cases.

at $(Q-q)=(1 / 4,1 / 4,0)$ is much less efficient than for the wave in Fig. 11. This is because of the high local DOS, and low band dispersion, near the $X$-point $(1 / 2,0,0)$. Therefore, the wave makes easily a gap near this point, where the coupling is large. The calculated $T_{C}$ is small. By including all phonons in the estimation of $\lambda=N \vartheta^{2} / M \omega^{2} u^{2} \approx 0.06$ (from these calculations $N=0.6 \mathrm{eV} / \mathrm{Cu} / \mathrm{spin}, \vartheta=0.07 \mathrm{eV}$ for $\mathrm{u}=0.07 \AA$, and using $M \omega^{2}=10 \mathrm{eV} / \AA^{2}$ for planar oxygens [5, 36, 37]) and $T_{C}$ is less than $1 K$. The FS at optimal doping is wide near $(1 / 2,0,0)$, where the Fermi velocity is low [38], and becomes narrower when $k$ is approaching $(1 / 4,1 / 4,0)$ because of larger band dispersion. The FS and states within $E_{F} \pm 0.2 \mathrm{eV}$ occupy not more than $1 / 4$ th of the BZ. With only $1 / 4$ of the elastic energy, $M \omega^{2} u^{2}$, from the efficient phonons near the X-point, $T_{C}$ becomes of the order 15 $K$. Higher values are obtained if spin fluctuations and SPC are included [6], but all these estimations are very approximate. It can be noted that $T_{C}$ in $\mathrm{Ba}_{2} \mathrm{CuO}_{3}$ is reported to be much larger than in $\mathrm{La}_{2} \mathrm{CuO}_{4}$ [39 41]. A possible reason could be that fewer apical oxygens make the interplane interaction weaker, so that the FS becomes even narrower than in $\mathrm{La}_{2} \mathrm{CuO}_{4}$ [42, 43]. 


\section{B. Disorder and fluctuations.}

There is a negative influence from thermal disorder on the well-defined modes that convey superconductivity. Two sets of calculations are made with disorder, where $u$ are 0.034 and $0.052 \AA$, corresponding to ZPM and at RT respectively. These distortions agree with isotropic values on $\mathrm{La}$ and $\mathrm{Cu}$ in undoped $\mathrm{La}_{2} \mathrm{CuO}_{4}$ as measured by Häfliger et al [44, while the measured values for O-sites are slightly larger. The measured distortion of planar $\mathrm{O}$ in the direction of $\mathrm{Cu}$ (the most important for the band gap at $\mathrm{X}$ ) is similar to what is used here. Our calculated broadening $(\Delta \epsilon)$ of the paramagnetic bands is on the average $18 \mathrm{meV}$ from ZPM and about $25 \mathrm{meV}$ at RT, and about twice as large in spin-polarized calculations, see Table I. Photoemission by Kondo et al 45 observed broadenings for states going from 0 to $13.5^{\circ}$ from the diagonal "node" direction on the FS, to be 6-9 meV at low $\mathrm{T}$ and about $22 \mathrm{meV}$ at $175 \mathrm{~K}$. Extrapolation towards states further away from the diagonal (and towards RT) would probably give larger values in better agreement with the calculated band broadening. For comparison we note that spectroscopic broadenings at RT in Li and $\mathrm{Na}$ are much larger, $150-180 \mathrm{meV}$ [23, 46]. This is because those alkali metals have very soft lattices so that the thermal disorder is very large when $T$ is close to the melting temperature. The average $u$ would be close to 10 percent of interatomic distances at melting according to the Lindemann rule [47.

A first observation is that the effective hole doping on $\mathrm{Cu}$ sites diminishes with disorder. The average valence charge on $\mathrm{Cu}, 10.35$ el., goes up by about 0.03 el. and $0.06 \mathrm{el}$. in the ZPM and RT results, respectively. These values are quite stable to spin polarization. But other effects of disorder are more important for the cuprate properties at high $T$.

TABLE I: Average magnetic moments on $\mathrm{Cu}\left(m_{a v}\right)$, averaged band broadening ( $\Delta \epsilon$, relative to ordered configurations) and maximal band deviations $\left(\epsilon_{\max }\right)$ for different configurations. The latter is located at k-points near the $X$-point (and caused by phonon/spin waves), where the DOS is highest, and can be interpreted as half of the gap value $\left(\frac{1}{2} E_{g}\right)$. The values of $\Delta \epsilon$ for the 3 first columns are within parentheses since they are enhanced by the gap near the X-point. The values of $\epsilon_{\max }$ for the 2 last columns are within parentheses because they are partly enhanced by disorder. Energies in meV.

\begin{tabular}{lccccc}
\hline & P-phon & AF-ord & AF+phon & AF-ZPM & AF+phon-RT \\
\hline \hline$m_{a v}$ & - & 0.192 & 0.217 & 0.186 & 0.154 \\
$\Delta \epsilon$ & $(20)$ & $(40)$ & $(60)$ & 35 & 50 \\
$\epsilon_{\max }$ & 50 & 70 & 85 & $(70)$ & $(80)$ \\
\hline
\end{tabular}

Fig. 2 shows the absolute values of the local moments on $\mathrm{Cu}$ in the ordered and disordered (at ZPM) supercells from spin-polarized calculations with identical applied AFM fields. The AFM wave is perfectly developed

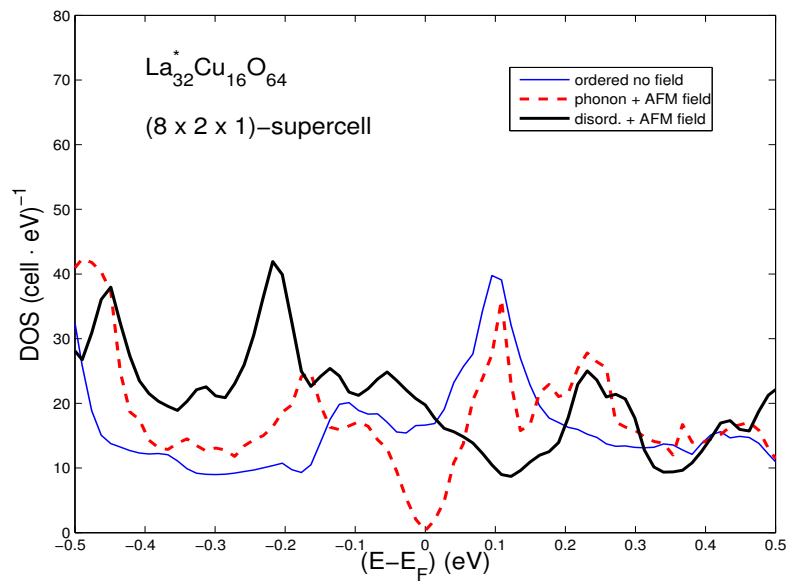

FIG. 3: (Color online) Unbroadened DOS functions for the non-magnetic undistorted supercell (thin blue line), for the cell with a "breathing planar oxygen" phonon and AFM fields (bold broken red line), and for one disordered cell with phonon+AFM fields (bold black line). The AFM fields induce stripes as in Fig. 1 The La* atoms have charge 56.875 in these virtual crystal calculations.

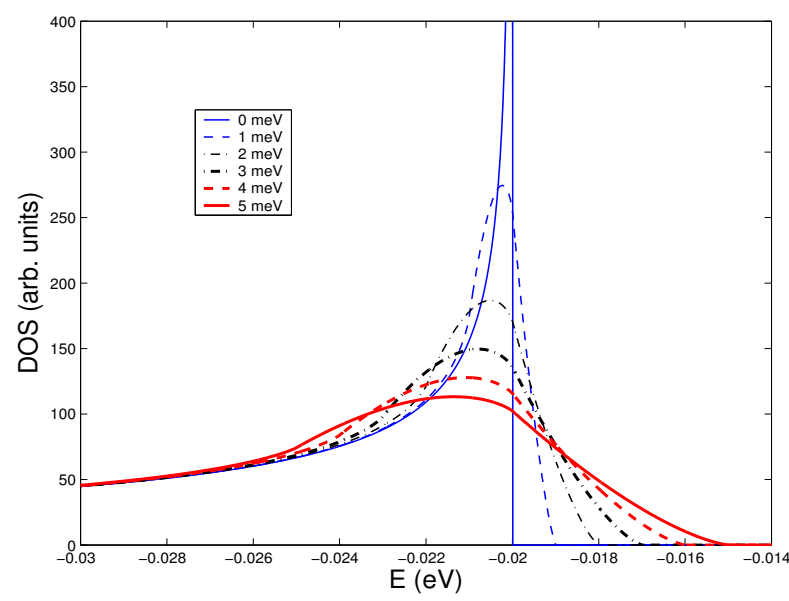

FIG. 4: (Color online) Schematic low-energy edge of the BCSlike band gap as function of disorder.

in the ordered cell with only three values of local moment amplitudes; the four sites at node positions have no moment, the four sites at the interior of the stripes have the largest, and the eight remaining ones have slightly smaller moments. This distribution of moments becomes more irregular for increasing disorder. At the AFM nodes (Cu without field) small moments develop with disorder. It is seen that the average moment decreases with disorder for long-wave spin modulations and for large moments despite the positive contributions of $\frac{d m}{d T}$ that always come from the node sites. This is also shown in Table II even when both spin waves and phonons are present.

Differently, results for shorter waves (in cells extending $4 a_{0}$ and $6 a_{0}$ along $\vec{x}$ ) show that disorder can enforce 
weak local moments. This is similar to what is found for disordered Cerium 22] compared to ordered fcc Ce [48, but only at the volumes near the onset of magnetism. A local volume expansion can increase the local moment, whereas a local compression cannot decrease an already weak moment, and on the average there is an increase 22. Overdoped cuprates are weakly magnetic and they have positive values of $\frac{d m}{d T}$, at least for $m$ up to 0.4 $\mu_{B} / \mathrm{Cu}$. A positive average of $\frac{d m}{d T}$ can also be found in weak moment configurations with long spin waves due to strong contributions from node sites. Not however that disorder destroys coherent waves in all cells, and the gaps at $E_{F}$ are more and more difficult to detect at ZPM and $\mathrm{RT}$ in the cells of different lengths and different average moments.

The effect of SPC, demonstrated by the higher moment in a lattice with a phonon distortion compared to the moment in the ordered structure, is weaker in the disordered lattices. This is rather independent of doping.

The coupling constant for SF appears to be more sensitive to disorder than the coupling for phonons, because the "clean" AFM wave suffers a lot in the disordered lattice. Instead of having a potential perturbation given by one Fourier coefficient at a precise $q$, there will be several coefficients, each one working to open up gaps at different parts of the band. This has a direct negative impact on the gap. The maximal band deviation, $\epsilon_{\max }$, is found at k-points near the position of the downfolded $X$-point, i.e. in the region of the $\mathrm{BZ}$ where the single phonon/spin wave opens up a gap. The two disordered configurations have large band broadenings at all k-points. On the contrary, at k-points where a well-defined wave makes a gap, disorder tends to wipe out the gap. In fact, $\epsilon_{\max }$ is not revealing for the latter cases, since it might be boosted from disorder just at one k-point.

The closing of the gap in the disordered lattice is seen from the DOS functions in Fig 3 . The thin line shows the DOS for the non-distorted non-magnetic supercell. The $N\left(E_{F}\right)$ is quite high since $E_{F}$ is close to the van-Hove singularity for this hole doping, although the DOS calculated from the limited number of k-points is not smooth. With the phonon and the spin-wave along the cell there is a gap and $N\left(E_{F}\right)=0$, as shown by the broken line. This DOS curve changes to the bold line when all atomic positions are disordered, and there is no sign of a gap any longer. Peaks and valleys in the DOS appear at other energies for this particular (randomized) disorder, but the superimposed DOS from several different disordered configurations becomes more smooth.

The next step is to project the parameters of band broadening from the ab-initio results on to the BCS DOS function at different $T$ in order to extract the effect of disorder on the DOS peak, on the superconducting gap $(\Delta)$ and on $T_{C}$. This is exemplified in Fig. 4 The DOS peak below $E_{F}$ becomes broadened, but its energy is essentially unchanged. The upper band edge will reach the lower band edge above the gap when $\Delta$ decreases at higher $T$. Superconductivity disappears approximately when $\Delta-\Delta \epsilon=0$, although it could survive longer through a mechanism of percolation. Small disconnected islands of fluctuating superconductivity can appear temporarily, and still produce a DOS-peak below a pseudogap. The gap defined from the peak in scanning tunneling microscopy (STM) can be larger than $50 \mathrm{meV}$ in underdoped cuprates with the highest $T_{C}$ 's, and the gap ratio $\left(2 \Delta / k_{B} T_{C}\right)$ is typically much larger than the BCSvalue (3.5) even in cuprates with lower $T_{C}$ [49. Suppose that $\Delta \approx 40 \mathrm{meV}$ at low $T$, as often found from STM for cuprates 49. This corresponds to a $T_{C}$ near $250 \mathrm{~K}$ according to BCS theory. The ZPM makes the DOSpeak broad. In the spin-polarized results for the supercell we have a broadening of about $30 \mathrm{meV}$ from ZPM, which makes a reduction of the band edge to $\sim 10 \mathrm{meV}$. At $150 \mathrm{~K}$, when a normal BCS gap would be reduced to $30-35 \mathrm{meV}$, the broadening increases to about $40 \mathrm{meV}$. The gap would be closing at $\sim 120 \mathrm{~K} \approx 11 \mathrm{meV}$, which would be the corrected $T_{C}$ in this example. Therefore, thermal disorder will reduce $T_{C}$ more than gap (defined from the DOS peak at $0 \mathrm{~K}$ ), which leads to a larger gapratio than in BCS. In the example above it would be 7.5, and the DOS-peak remains visible for $T>T_{C}$. Obviously, these results are only qualitative, but the general conclusions are; ${ }^{1}$ that thermal disorder can produce superconducting fluctuations and a pseudogap above $T_{C}$, ${ }^{2}$ that the gap-ratio is larger than the BCS value in underdoped cuprates, and ${ }^{3}$ that a higher $T_{C}$ is expected if disorder could be suppressed.

Band calculations show that the coupling parameter for spin-fluctuations goes up for decreased doping (more than for phonons), and it tends to diverge before static AFM makes the material insulating [ [5]. Therefore, it is on the underdoped side of the phase diagram that we can expect the strongest disorder effects on superconductivity. There is a competition between large possible $T_{C}$ and its destruction from disorder, because both mechanisms depend on the coupling parameter for spin fluctuations. The absence of superconductivity at low $T$ and very low doping can be because the increased coupling makes disorder important already from ZPM, while this is not the case at higher doping. This makes sense since the effect of disorder is accumulated over a long distance and therefore more destructive for long waves at low doping. Another possibility is that the increasingly long wavelengths of the modulations at low doping make superconductivity sensitive to the short coherence length [15]. Long-range superconductivity is replaced by fluctuations in both cases.

\section{Making $T_{C}$ resistent to disorder?}

The simple relations of how $\lambda$ and $T_{C}$ depend on $N$, $\vartheta$ and $K u^{2}$, indicate ways for increasing $T_{C}$ if disorder plays no role. A different view of the high- $T_{C}$ problem, and how to increase $T_{C}$, is possible if it turns out that the pseudogap at low doping is caused by superconducting 
fluctuations in a thermally disordered lattice. One could then recuperate a higher $T_{C}$ through stabilization of existing superconducting fluctuations, but it is difficult to get rid of thermal disorder. Larger coupling constants for spin fluctuations should be efficient to enforce the "clean" spin waves. However, a large $\vartheta_{m}$ would also enforce fluctuations and bad influence from disorder could spoil the effect of enforced spin waves. Structural disorder will be smaller in a stiffer lattice at a given $T$, and since the spin disorder is largely an effect of the structural disorder, it suggests that lattice hardening would help. This can be achieved by applying pressure, $P$, as long as the strength of the spin wave is preserved. A higher $N\left(E_{F}\right)$ leads to a larger gain in electronic energy from a superconducting gap, and $T_{C}$ would be higher according to eq. 3 . This is also valid at large disorder. Broadening of DOS peaks, which are not exactly at $E_{F}$ at low $T$, can be favorable for increasing $N\left(E_{F}\right)$ at large $T$. Another way to increase $N$ is to introduce new (static) potential modulations in the lattice $[28$ 30]. Such modulations could be caused by (natural or artificial) dopant orderings, which make peaks in the DOS at $E_{F}$ if the period is correct 31. In this case we imagine that the FS is not going to be more complicated, but that the band at $E_{F}$ becomes more flat. Even efforts to decrease $\vartheta_{m}$ in order to avoid static AFM, or suppress AFM fluctuations, in the very underdoped region could have a positive effect on $T_{C}$. This can be achieved by applied pressure. In fact, $d T_{C} / d P$ appears to be positive more so at low doping than in the overdoped region [50, even if other $P$-dependent variations (phase transitions, charge carrier transfers within the structure) can also modify $T_{C}$.

\section{CONCLUSION.}

The conclusion from these results is that the effective $\lambda^{\prime} s$ can larger than what is generally believed. This is because the superconducting mechanism depend on few phonon/spin excitations. However, gaps and long-range phonon/spin waves are sensitive to disorder and there is a limitation of long-range superconductivity and $T_{C}$ because of thermal disorder. This is most likely at low doping where the coupling from spin-fluctuations is largest. A softened lattice may increase $\lambda$, but it also makes the amplitude of disorder larger. To increase $N\left(E_{F}\right)$ by letting $E_{F}$ to be on a DOS-peak should increase the effective $\lambda$ even if $\vartheta$ is constant. This could therefore be a way to reach a higher $T_{C}$ through a moderation of the harmful effects from thermal disorder.

Acknowledgement I am grateful to A. Bianconi and C. Berthod for useful discussions.
[1] W.L. McMillan, Phys. Rev. 167, 331, (1968).

[2] W. Kohn and L.J. Sham, Phys. Rev. 140, A1133 (1965).

[3] M. Dacorogna, T. Jarlborg, A. Junod, M. Pelizzone and M. Peter, J. Low Temp. Phys. 57, 629, (1984).

[4] G.D. Gaspari and B.L. Gyorffy, Phys. Rev. Lett. 28, 801, (1972).

[5] T. Jarlborg, Phys. Rev. B76, 140504(R), (2007).

[6] T. Jarlborg, Physica C454, 5, (2007).

[7] T. Jarlborg, Physica C385, 513, (2003).

[8] T. Jarlborg, Phys. Lett. A300, 518 (2002).

[9] J. Bardeen, L.N. Cooper and J.R. Schrieffer, Phys. Rev. 108, 1175 (1957).

[10] T. Jarlborg, Solid State Commun. 151, 639, (2011).

[11] T. Jarlborg, Solid State Commun. 181, 15, (2014).

[12] G. Santi, T. Jarlborg, M. Peter and M. Weger, Physica C 259, 253, (1996) DOI: 10.1016/0921-4534(96)00052-4

[13] E.G. Moroni and T. Jarlborg, Phys. Rev. B 41, 9600, (1990).

[14] Y. Y. Peng, M. Hashimoto, M. Moretti Sala, A. Amorese, N. B. Brookes, G. Dellea, W.-S. Lee, M. Minola, T. Schmitt, Y. Yoshida, K.-J. Zhou, H. Eisaki, T. P. Devereaux, Z.-X. Shen, L. Braicovich and G. Ghiringhelli, arXiv:1504.05165v1, (2015).

[15] T. Jarlborg, J. of Supercond. and Novel Magn., 28, 1231, (2014) DOI: 10.1007/s10948-014-2897-1

[16] T. Jarlborg, Phys. Rev. B59, 15002, (1999).

[17] T. Jarlborg, Phys. Rev. Lett. 77, 3693, (1996).

[18] T. Jarlborg, Phys. Lett. A 236, 143, (1997).

[19] P. Pedrazzini, H. Wilhelm, D. Jaccard, T. Jarlborg, M. Schmidt, M. Hanfland, L. Akselrud, H.Q. Yuan, U.
Schwarz, Yu. Grin and F. Steglich, Phys. Rev. Lett. 98, 047204, (2007).

[20] O. Delaire, K. Marty, M.B. Stone, P.R. Kent, M.S. Lucas, D.L. Abernathy, D. Mandrus, and B.C. Sales, PNAS 108, 4725, (2011).

[21] T. Jarlborg and G. Santi, Physica C 329, 243, (2000).

[22] T. Jarlborg, Phys. Rev. B89, 184426 (2014).

[23] L. Hedin and A. Rosengren, J. Phys. F: Metal Phys. 7, 1339, (1977).

[24] R.H. McKenzie and J.W. Wilkins, Phys. Rev. Lett. 69, 1085, (1992).

[25] T. Jarlborg, Phys. Rev. B76, 205105, (2007).

[26] T. Jarlborg, P. Chudzinski and T. Giamarchi, Phys. Rev. B85, 235108, (2012).

[27] S.B. Dugdale and T. Jarlborg, Solid State Commun. 105, 283, (1998).

[28] T. Jarlborg and A. Bianconi, Phys. Rev. B 87, 054514, (2013) DOI: 10.1103/PhysRevB.87.054514

[29] N. Poccia, M. Fratini, A. Ricci, G. Campi, L. Barba, A. Vittorini-Orgeas, G. Bianconi, G. Aeppli, A. Bianconi, Nature Materials, 10, 733 (2011).

[30] O. Chmaissem, I. Grigoraviciute, H. Yamauchi, M. Karppinen and M. Marezio, Phys. Rev. B 82, 104570, (2010).

[31] T. Jarlborg, Appl. Phys. Lett. 94, 212503, (2009).

[32] T. Jarlborg and A. Bianconi, (unpublished) (2015).

[33] O.K. Andersen, Phys. Rev. B12, 3060 (1975).

[34] B. Barbiellini, S.B. Dugdale and T. Jarlborg, Comput. Mater. Sci. 28, 287 (2003).

[35] O. Gunnarsson and B.I Lundquist, Phys. Rev. B 13, 4274 (1976). 
[36] T. Jarlborg, J. Phys. Condens. Matter 16, L173 (2004).

[37] T. Jarlborg, Phys. Rev. B79, 094530, (2009).

[38] T. Jarlborg, Phys. Rev. B84, 064506, (2011).

[39] Q. Q. Liu, H. Yang, X. M. Qin, Y. Yu, L. X. Yang, F. Y. Li, R. C. Yu, and C. Q. Jin, Phys. Rev. B 74, 100506, (2006).

[40] W.B. Gao, Q.Q. Liu, L.X. Yang, Y. Yu, F.Y. Li, C.Q. Jin and S. Uchida, Phys. Rev. B 80, 094523 (2009).

[41] T.H. Geballe and M. Marezio, Physica C 469, 680, (2009).

[42] T. Jarlborg, B. Barbiellini, R.S. Markiewicz and A. Bansil, Phys. Rev. B 86, 235111, (2012) DOI: 10.1103/PhysRevB.86.235111.

[43] T. Jarlborg, A. Bianconi, B. Barbiellini, R.S. Markiewicz, A. Bansil, J. Supercond. Nov. Magn. 26, 2597, (2013) DOI $10.1007 / \mathrm{s} 10948-013-2142-3$

[44] P. S. Häfliger, S. Gerber, R. Pramod, V. I. Schnells, B. dalla Piazza, R. Chati, V. Pomjakushin, K. Conder, E. Pomjakushina, L. Le Dreau, N. B. Christensen, O. F. Syljuåsen, B. Normand, and H. M. Rønnow, Phys. Rev. B89, 085113 (2014).

[45] T. Kondo, W. Malaeb, Y. Ishida, T. Sasagawa, T. Tohyama, and S. Shin, arXiv:1404.0911, (2014).

[46] Y. Baer, P.H. Citrin and G.K. Wertheim, Phys. Rev. Lett. 37, 49, (1976).

[47] G. Grimvall. Thermophysical Properties of Materials (North-Holland, Amsterdam, 1986).

[48] T. Jarlborg, E.G. Moroni and G. Grimvall, Phys. Rev. B 55, 1288, (1997).

[49] Ø. Fischer, M. Kugler, I. Maggio-Aprile, C. Berthod and C. Renner, Rev. Mod. Phys., 79, 353, (2007), (and refs. given therein).

[50] J.S. Schilling, cond-matt/0604090, (2006). 